\title{
Genetic Analyses of DNA Methyltransferases in Arabidopsis thaliana
}

\author{
X. ZHANG* AND S.E. JACOBSEN*广 \\ *Department of Molecular, Cell and Developmental Biology, ${ }^{\dagger}$ Howard Hughes Medical Institute, \\ University of California, Los Angeles, California 90095
}

\begin{abstract}
DNA methylation is a conserved epigenetic silencing mechanism that functions to suppress the proliferation of transposons and regulate the expression of endogenous genes. In plants, mutations that cause severe loss of DNA methylation result in reactivation of transposons as well as developmental abnormalities. We use the flowering plant Arabidopsis thaliana as a model system to study the establishment and maintenance of DNA methylation as well as its role in regulating plant development. The genetic evidence presented here suggests that methylation at CG and non-CG sites functions in a partially redundant and locus-specific manner to regulate a wide range of developmental processes. Results from recent studies also suggested that the dynamic nature of non-CG methylation, which is critically important for its regulatory function, is largely due to its complicated interactions with other epigenetic pathways such as RNAi and histone modifications. Finally, the use of genomic approaches has significantly broadened our understanding of the patterning of DNA methylation on a genomewide scale and has led to the identification of hundreds of candidate genes that are controlled by DNA methylation.
\end{abstract}

Most eukaryotic organisms modify their genomic DNA in certain regions of the genomes through the addition of a methyl group to the $\mathrm{C} 5$ position of cytosine residues. This process is commonly referred to as "DNA methylation," and it is the most prevalent type of DNA modification in eukaryotes. DNA methylation is an important epigenetic silencing mechanism, as genomic regions that are densely methylated often correlate with reduced transcriptional activity. For this reason, as well as the observation that the repetitive fractions of eukaryotic genomes are often methylated, DNA methylation is generally thought to have evolved initially as a defense mechanism against transposons or other types of invading DNA (Yoder et al. 1997; Martienssen and Colot 2001). However, it has also been "domesticated" by the genome to regulate the expression of endogenous genes and became critically important for normal development. For example, null mutations in the maintenance methyltransferase DNMT1 or the de novo methyltransferase DNMT3a/b genes cause embryonic lethality in mouse, and the loss of DNMT1 function in a human cell line leads to reduced cell viability (Goll and Bestor 2005; Egger et al. 2006). Similarly, severe loss of DNA methylation in plants leads to several developmental abnormalities (Chan et al. 2005).

In mammals, DNA methylation is established and maintained mostly at CG sites through the action of the DNA methyltransferases DNMT1 and DNMT3a/b (Bird 2002; Goll and Bestor 2005). In contrast, plant genomes contain DNA methylation in all sequence contexts (Chan et al. 2005). The plant DNMT1-homolog METHYLTRANSFERASE 1 (MET1) maintains DNA methylation at CG sites (CG methylation) (Finnegan et al. 1996; Ronemus et al. 1996; Kankel et al. 2003; Saze et al. 2003), whereas the DNMT3a/3b homolog DOMAINS REARRANGED METHYLASE 1 and 2 (DRM1/2) are responsible for the de novo methylation in all sequence contexts as well as for the maintenance methylation at asymmetric $\mathrm{CHH}$ sites (where $\mathrm{H}=\mathrm{A}, \mathrm{C}$, or $\mathrm{T}$; $\mathrm{CHH}$ methylation) (Cao and Jacobsen 2002b). In addition, the plant-specific CHROMOMETHYLASE3 (CMT3) is responsible for DNA methylation at $\mathrm{CNG}$ sites $(\mathrm{CNG}$ methylation), as well as a subset of $\mathrm{CHH}$ sites (Bartee et al. 2001; Lindroth et al. 2001).

The added complexity of DNA methylation in plants compared to animals is reflected not only in the additional sequence contexts and the methyltransferase CMT3, but also in the intimate relationship and complicated interplay between DNA methylation and RNAi as well as histone H3 methylation. Components of the RNAi and histone methylation pathways are required for the establishment and maintenance of proper DNA methylation patterns. Conversely, some mutations that result in the partial loss of DNA methylation also affect the functions of the RNAi pathway and histone methylation (Chan et al. 2005). Thus, DNA methylation, RNAi, and histone methylation function together to maintain the proper function of plant genomes.

Here, we describe the results of our genetic and genomic analyses of DNA methylation in Arabidopsis. Analyses of multiple mutants that lack DNA methylation in different combinations of sequence contexts suggested that $\mathrm{CG}, \mathrm{CNG}$, and $\mathrm{CHH}$ methylation act in a partially redundant manner to regulate many aspects of normal plant development. We also discuss the complex interactions between DNA methylation, RNAi, and histone methylation. Finally, results from our genome-wide mapping of the sites of DNA methylation and transcriptional analysis of DNA methyltransferase mutants provided direct evidence for the critically important role of DNA methylation in silencing transposable elements and regulating endogenous genes. 


\section{DNA METHYLATION CONTROLS MANY ASPECTS OF ARABIDOPSIS DEVELOPMENT}

Severe loss of CG methylation in mutants such as met 1 and $d d m 1$ resulted in developmental abnormalities, suggesting that CG methylation is important in controlling endogenous gene expression (Finnegan et al. 1996; Ronemus et al. 1996; Jeddeloh et al. 1999; Kankel et al. 2003; Saze et al. 2003). In fact, a few genes have been found to be misregulated in met 1 and are responsible for some of the developmental phenotypes. The most common type of misregulation is likely due to derepression caused by the loss of DNA methylation. For example, the hypomethylation and ectopic overexpression of the $F W A$ gene in met1 result in late flowering (Soppe et al. 2000). A second type of misregulated genes is exemplified by SUPERMAN. The hypermethylation and silencing of the SUPERMAN gene cause several floral defects such as increased number of stamens, incompletely fused carpels, and partial sterility (Jacobsen and Meyerowitz 1997). This hypermethylation process is not yet well understood but appears to be dependent on DRM1/2 (X. Zhang et al., unpubl.).

In contrast, both $\mathrm{cmt} 3$ and $d r m 1 / 2$ mutants were found to be phenotypically normal even after prolonged inbreeding (Bartee et al. 2001; Lindroth et al. 2001; Cao and Jacobsen 2002b). This might suggest that non-CG methylation is dispensable for normal plant development. However, it soon became clear that DRM1/2 and CMT3 acted in a partially redundant and locus-specific manner, and only in the $d r m 1 / 2 \mathrm{cmt} 3$ triple mutant was the vast majority of non-CG methylation eliminated (Cao et al. 2003; Chan et al. 2006). Importantly, gross loss of nonCG methylation (but with little change in CG-methylation) leads to several developmental defects, such as twisted leaves, short stature, and partial sterility (Cao et al. 2003; Chan et al. 2006). Notably, loss of DRM1/2 and CMT3 activities in both the Landsberg erecta (Ler) and the Columbia (Col) ecotypes of Arabidopsis led to very similar phenotypes, indicating that some aspects of the function of non-CG methylation in regulating plant development are evolutionarily conserved (Cao et al. 2003; Chan et al. 2006).

These results clearly demonstrated the requirement for DNA methylation in normal plant development. They also suggested that two different sets of genes were misregulated in met 1 and $d r m 1 / 2 \mathrm{cmt} 3$, as these two mutants displayed very different developmental abnormalities. It is therefore possible that CG methylation and non-CG methylation regulate distinct aspects of plant development. Alternatively, there may be considerable functional redundancies between $\mathrm{CG}$ and non-CG methylation. We therefore tested this hypothesis by constructing and analyzing mutants defective in both CG and non-CG methylation (drm1/2 met1 and cmt3 met 1$)$ following the scheme shown in Figure 1A.

Both drm 1/2 met 1 triple and cmt3 met 1 double mutant plants could be recovered at low frequency from drm $1 / 2 /+$ met $1 /+$ and $c m t 3 /+$ met $1 /+$ parents, respectively. This result suggests that the loss of DRM1/2 and MET1 or CMT3 MET1 activities does not necessarily lead to gamete or embryo lethality. However, a severe defect in seed viability was observed for both mutants. This is more apparent in $\mathrm{F}_{1}$ populations from $d r m 1 / 2$ met $1 /+$ and $c m t 3$ met $1 /+$ parents, where instead of approximately $1 / 4$, only about $1 / 40$ and about $1 / 20$ plants were found to be drm1/2 met1 and cmt3 met1, respectively. Both drm 1/2 met 1 and cmt 3 met 1 adult plants exhibited a number of severe developmental defects, such as extremely late flowering, reduced leaf size, shorter stature, and complete sterility (Fig. 1B). In addition, the flowers of cmt3 met1 plants displayed an agamous-like phenotype and developed sepals and numerous petals, but completely lacked stamens or carpels. Indeterminate flowers were also frequently observed. In contrast, typical flowers from $d r m 1 / 2$ met 1 plants only had two (instead of four) sepals, completely lacked petals, and developed numerous stamens and abnormal carpels. Thus, the additional and more severe defects of drm 1/2 met1 and $\mathrm{cmt} 3$ met 1 compared to previously isolated mutants strongly supported the notion that CG methylation and non-CG redundantly control developmentally important genes in Arabidopsis. It is also possible that the gross loss of DNA methylation in these multiple mutants may undermine the general structure and function of the chromosomes (e.g., chromosome segregation or heterochromatin condensation) and thus affect normal cell divisions.

To characterize the changes in DNA methylation pattern in the $d r m 1 / 2$ met 1 and cmt3 met 1 mutants, we performed bisulfite genomic sequencing at selected loci. To this end, we bisulfite-sequenced the retroelement AtSN1 and an intergenic tandem repeat downstream of the $M E D E A$ gene (MEA ISR), both of which have been wellcharacterized in wild-type and several mutant plants (Cao and Jacobsen 2002a; Zilberman et al. 2003; Henderson et al. 2006). As shown in Figure 2, virtually all methylation was eliminated from cmt 3 met 1 at both loci. In contrast, in the drm1/2 met 1 mutant, methylation in all sequence contexts was undetectable at MEA ISR, but significant CNG methylation remained at AtSN1. Note that the DNA methylation patterns in $d r m 1 / 2$ met 1 and cmt 3 met 1 were different from all previously described single or multiple DNA methyltransferase mutants (Fig. 2) (Cao and Jacobsen 2002a; Zilberman et al. 2003; Henderson et al. 2006). Taken together, these results revealed complex interdependence of the DRM1/2, CMT3, and MET1 activities in the establishment and maintenance of DNA methylation, and provided further support for their functional redundancies in a locus-specific manner.

We also attempted to construct the quadruple mutant $\mathrm{drm} 1 / 2 \mathrm{cmt} 3 \mathrm{met} 1$, in which the vast majority of methylation (if not all) should be eliminated. We were unable to isolate $d r m 1 / 2 \mathrm{cmt} 3$ met 1 from $\mathrm{drm} 1 / 2 /+\mathrm{cmt3} /+$ met $1 /+$ parents, but $d r m 1 / 2 \mathrm{cmt} 3 \mathrm{met} 1 /+$ plants were readily obtained, indicating that the activities of DRM1/2, CMT3, and MET1 may be dispensable for male and/or female gamete development. However, inspection of developing siliques from $d r m 1 / 2 \mathrm{cmt} 3$ met $1 /+$ plants revealed that approximately $1 / 4$ of the seeds aborted at very early stages (Fig. 3), which were likely to be $d r m 1 / 2$ cmt3 met1. It therefore appears that the proper methylation at some loci is critical for early embryo development. 
Downloaded from symposium.cshlp.org on April 26, 2023 - Published by Cold Spring Harbor Laboratory Press

DNA METHYLATION PATTERNING IN A. THALIANA

A

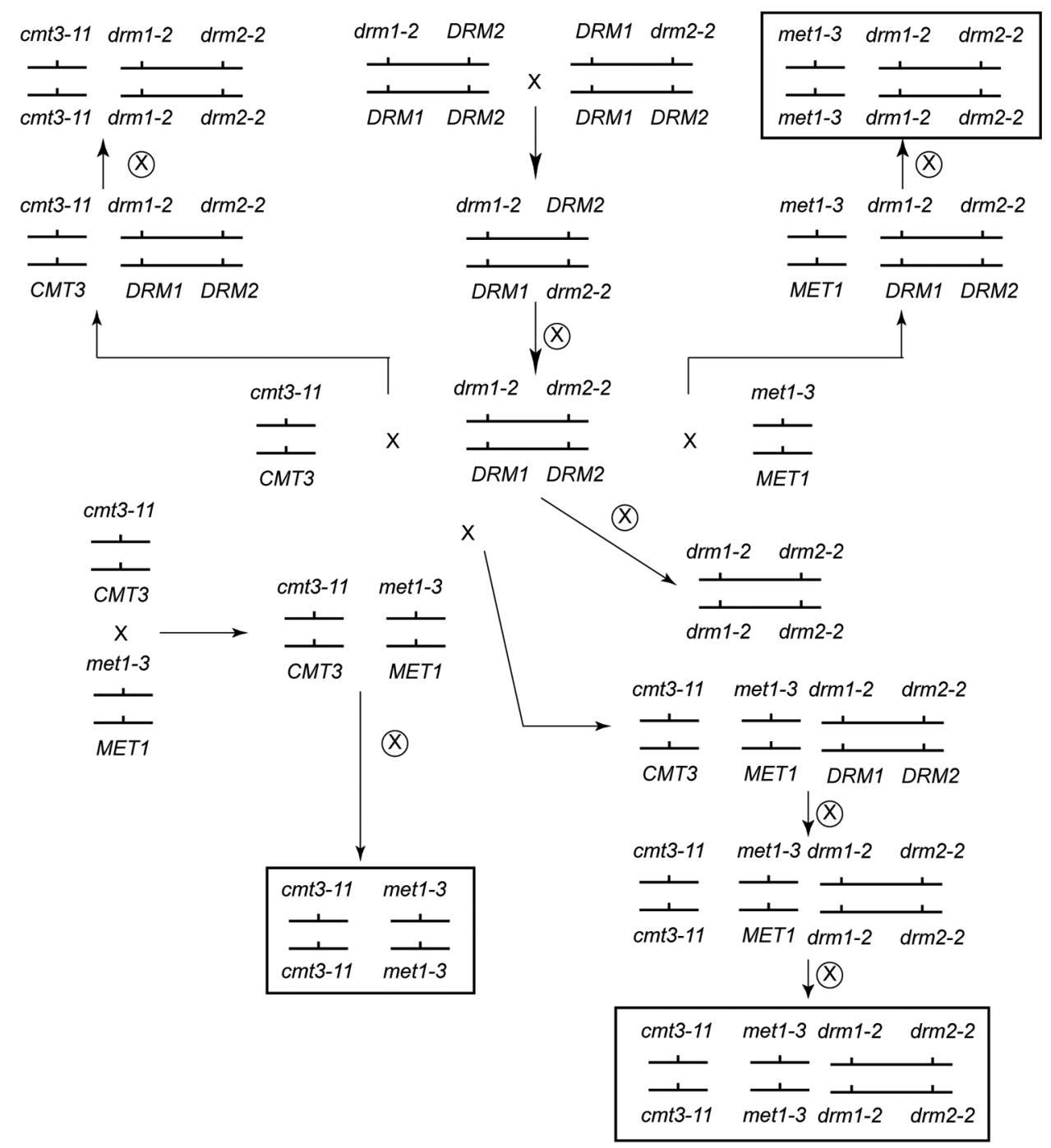

B
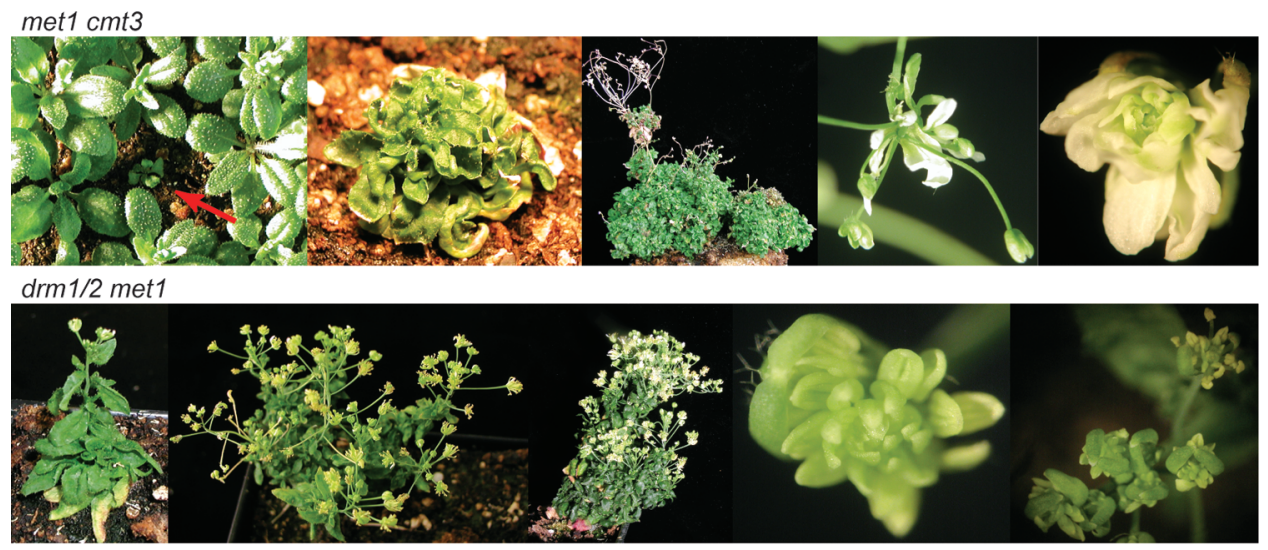

Figure 1. Partially redundant function of CG and non-CG methylation in regulating plant development. (A) Genetic schemes for constructing multiple mutants. $(B)$ Developmental phenotypes of cmt3 met 1 (top row) and drm 1/2 met1 (bottom row) are significantly more severe than either met 1 or $d r m 1 / d r m 2 ~ c m t 3$ null mutants (Cao et al. 2003; Saze et al. 2003; Chan et al. 2006). Red arrow indicates a met $1 \mathrm{cmt} 3$ plant among other plants which are not met $1 \mathrm{cmt} 3$ double mutants. 

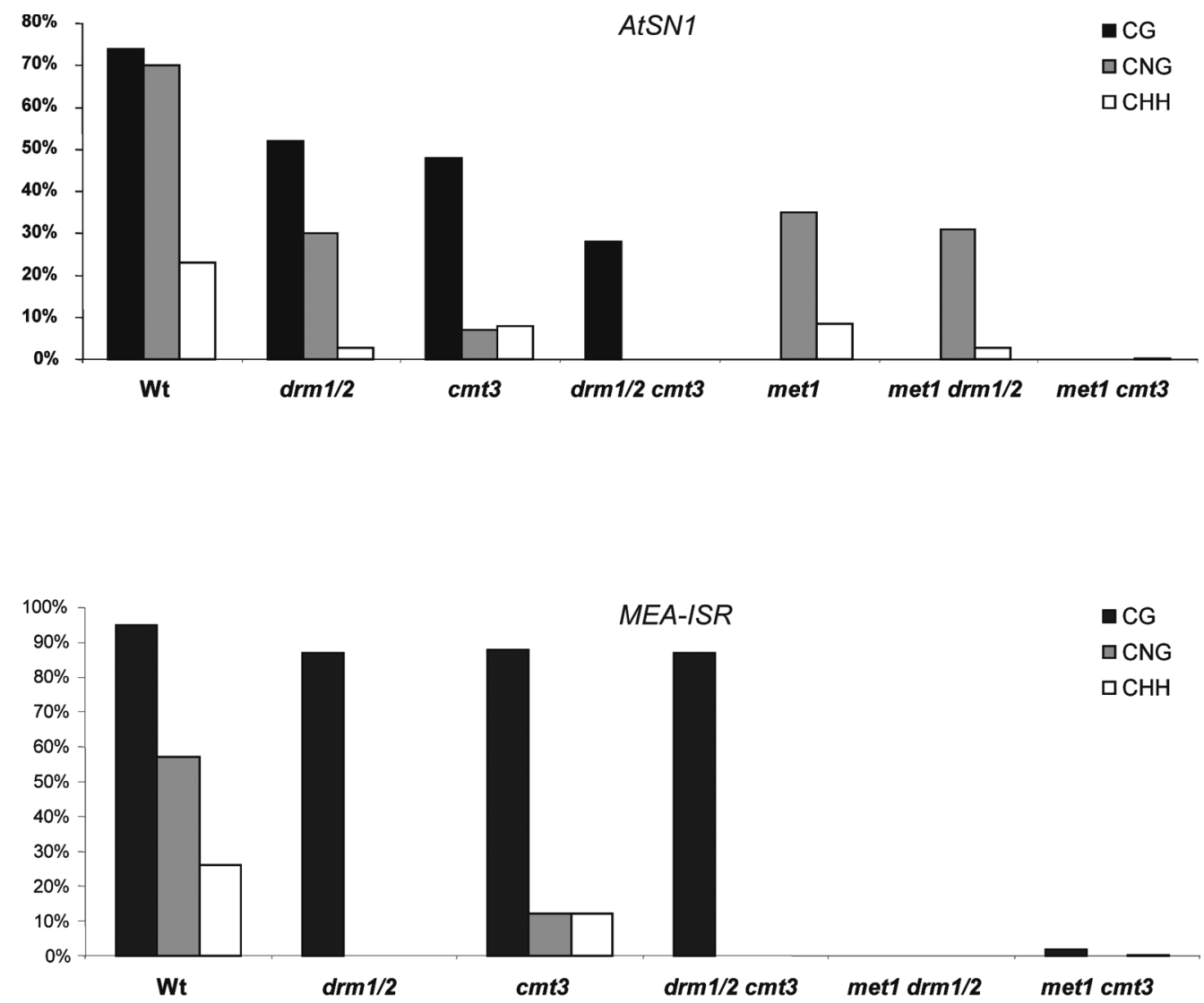

Figure 2. Severe loss of DNA methylation in $d r m 1 / 2$ met 1 and met $1 \mathrm{cmt} 3$. Bisulfite sequencing results of AtSN1 and MEA-ISR in these two mutants are compared to those from previously described DNA methyltransferase mutants (Zilberman et al. 2003; Henderson et al. 2006). Black, gray, and open bars represent the percentage of DNA methylation at CG, CNG, and CHH sites, respectively.

In extremely rare cases $(1$ in $\sim 1000)$, drm $1 / 2 \mathrm{cmt} 3$ met 1 plants were recovered from $d r m 1 / 2 \mathrm{cmt} 3$ met $1 /+$ parents. Such quadruple mutant plants grew very slowly, exhibited a suite of severe developmental phenotypes, and failed to flower after about 7 months (Fig. 3).

The results described above strongly suggested that the activities of MET1, DRM1/2, and CMT3 act redundantly to regulate gene expression. The severity of the phenotypes of drm1/2 met1, cmt3 met1, or drm1/2 cmt3 met1 plants also underscored the importance of DNA methylation in regulating early embryogenesis as well as many aspects of the normal development of Arabidopsis. However, these results still may not have revealed the full spectrum of the regulatory role of DNA methylation. For example, it is possible that the severe phenotypes of $\mathrm{drm} 1 / 2 \mathrm{met} 1, \mathrm{cmt} 3$ met 1 , or $d r m 1 / 2 \mathrm{cmt} 3$ met 1 could have masked the more subtle effects of the misregulation of many other genes. We derived a simple genetic scheme to test this possibility. A population of plants was created, in each of which a random subset of the chromosomes lacked MET1-dependent as well as DRM1/2- and/or CMT3-dependent methylation, whereas the rest of the chromosomes only lacked DRM1/2and/or CMT3-dependent methylation. It has been described that during the gametogeneses of met1/+ plants, the lack of MET1 activity in the met1 gametes resulted in chromosomal-level loss of DNA methylation following the
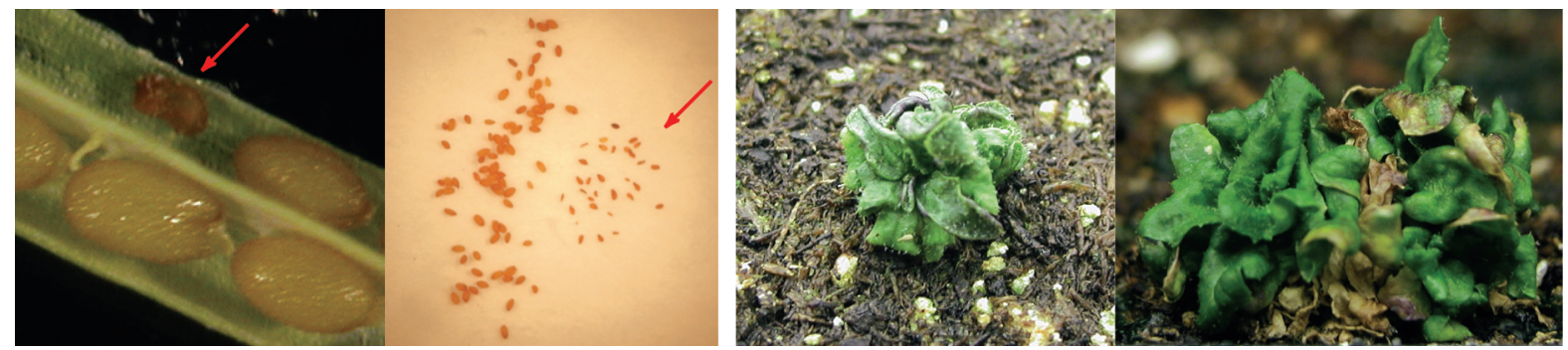

Figure 3. DNA methylation is required for embryogenesis and normal plant development. Approximately one-fourth of the seeds from drm1/2 cmt3 met1/+ plants abort at a very early developmental stage (left panels; red arrows). A rare $d r m 1 / 2 \mathrm{cmt} 3 \mathrm{met} 1 \mathrm{plant}$ displaying very delayed and stunted development is shown in the right panels. 
postmeiotic mitoses (two and three round of mitoses for male and female gametes, respectively) (Saze et al. 2003). Thus, in the mature male met 1 gamete, each chromosome has a $50 \%$ probability of being hemimethylated $(25 \%$ for a female met 1 gamete), and a 50\% probability of completely lacking MET1-dependent methylation (unmethylated; $75 \%$ for a female met 1 gamete). When combined with a wild-type gamete of the opposite sex to form a met1/+ zygote, DNA methylation on the hemimethylated chromosomes is immediately restored by the introduced MET1 activity, but the unmethylated chromosomes would remain unmethylated because the methylation pattern has largely been erased. Numerous genes located on such unmethylated chromosomes would be hypomethylated and some misregulated, thus resulting in a metl-like phenotype (albeit milder) in met $1 /+$ plants. We self-pollinated drm $1 / 2$ met $1 /+, \mathrm{cmt} 3 \mathrm{met} 1 / \mathrm{+}$, and $d \mathrm{rm} 1 / 2 \mathrm{cmt} 3 \mathrm{met} 1 /+$ plants and examined the $F_{1}$ plants that were heterozygous for MET1. These plants entirely lacked DRM1/2 and/or CMT3 activities. In addition, as described above, a random subset of their chromosomes also lacked MET1-dependent methylation. A remarkably variable assortment of developmental phenotypes were observed among $\operatorname{drm} 1 / 2$ met $1 /+$ or drm 1/2 cmt3 met1/+ plants, ranging from mild to extremely severe (Fig. 4). This was likely due to the misregulation of different sets of genes in individual plants, because they were deficient for DRM1/2 and/or CMT3 as well as MET1-dependent methylation for different combinations of chromosomes. In addition, similar phenotypes were found to arise in multiple independent plants. This is consistent with the reproducible misregulation of specific genes controlling these phenotypes and suggests that the stochastic mutagenic effect of transposon reactivation is not a major factor. Thus, a large number of genes important for many aspects of Arabidopsis development are regulated by DNA methylation. Interestingly, cmt3 met1/+ plants appeared phenotypically similar to met $1 /+$ and did not exhibit additional defects. Among other possibilities, it is interesting to consider that the molecular lesion responsible for the cmt 3 met 1 phenotype might be recessive (similar to the superman epimutation in met 1 ) or that the simultaneous misregulation of several genes located on different chromosomes might be required to render the phenotype.

\section{RNAI AND HISTONE METHYLATION IN THE ESTABLISHMENT AND MAINTENANCE OF NON-CG METHYLATION}

As a regulatory mechanism for gene expression and thus plant development, DNA methylation itself is likely to be highly regulated and able to respond to developmental and environmental cues. Several studies using a methylation-sensitive amplified fragment length polymorphism (AFLP) method have provided evidence that changes in DNA methylation may occur during plant development or under stress conditions (Messeguer et al. 1991; Finnegan et al. 1998; Xiong et al. 1999; Zluvova et al. 2001; Bitonti et al. 2002; Fraga et al. 2002; RuizGarcia et al. 2005). MET1-mediated CG methylation is remarkably stable across multiple generations. In contrast, non-CG methylation is much more dynamic and thus more appealing as a regulatory mechanism. This is because the establishment and maintenance of both DRM1/2-dependent CNN and CMT3-dependent CNG methylation require persistent targeting by the RNAi and histone methylation pathways, respectively.

DRM1/2 is required to establish de novo DNA methylation on an unmethylated incoming transgene (Cao and Jacobsen 2002b). To identify other upstream components of this pathway, a reverse genetic approach was undertaken where a collection of mutants defective in various epigenetic pathways were transformed with the gene $F W A$. The transgene was methylated and silenced in wildtype but not in the $d r m 1 / 2$ mutant, resulting in a late-flowering phenotype (Cao and Jacobsen 2002b). Several mutants defective in the RNAi pathway, including nrpdla, $n r p d 1 b, d r d 1, r d r 2, d c l 3$, and ago4, were found to resemble $d r m 1 / 2$ in that they blocked the de novo methylation of the FWA transgene (Dalmay et al. 2000; Zilberman et al. 2003; Chan et al. 2004, 2006; Kanno et al. 2005; Pontier et al. 2005). Furthermore, these mutants phenocopied drm1/2 in that they lost significant amounts of non-CG methylation at several endogenous loci tested (Hamilton et al. 2002; Chan et al. 2004, 2006; Herr et al. 2005; Kanno et al. 2005; Onodera et al. 2005; Pontier et al. 2005). These results suggested that the RNAi pathway is required for all aspects of the DRM1/2 activity. Importantly, these studies also provided a mechanism by which DRM1/2-dependent DNA methylation can be regulated in a tissue- and locusspecific manner through the activities of the RNAi pathway. Indeed, a recent deep sequencing analysis of Arabidopsis small RNAs revealed profound differences in the abundance and diversity of the small RNAs accumulated in different tissues (Lu et al. 2005).

Some RNAi genes such as NRPD1a/1b and NRPD $2 a / 2 b$ are plant-specific and have likely evolved only in the plant lineage (Herr et al. 2005; Kanno et al. 2005; Onodera et al. 2005; Pontier et al. 2005), whereas other genes such as RDRs, DCLs, and ARGONAUTEs are conserved but have undergone extensive duplications and functional diversifications in plants. As a result, the complexity of plant RNAi pathways far exceeds that of their animal counterparts. For example, the four Arabidopsis DCL genes are functionally specialized in the metabolism of different classes of small RNAs: DCL1 is required in the microRNA (miRNA) pathway, DCL3 is required for small interfering RNAs (siRNA), both DCL1 and DCL4 are required for trans-acting siRNAs (tasiRNA), and DCL1 and DCL2 are required for siRNAs derived from natural antisense transcripts (natsiRNA) (Schauer et al. 2002; Xie et al. 2004, 2005; Borsani et al. 2005; Dunoyer et al. 2005; Gasciolli et al. 2005; Yoshikawa et al. 2005; Henderson et al. 2006). It is of particular interest to determine whether all these pathways can interact with DRM1/2 to direct DNA methylation and transcriptional gene silencing. To address this question, we constructed all pair-wise combinations of double mutants between $d c l 2, d c l 3$, and $d c l 4$ as well as the triple mutant $d c l 2 d c l 3 d c l 4$ (Henderson et al. 2006). Comparison of the small RNA populations from wild type and $d c l 2 d c l 3 d c l 4$ by large-scale 454 sequencing showed that DCL1 alone was not only sufficient for all aspects of miRNA metabolism, but also capable of generating 21-bp 


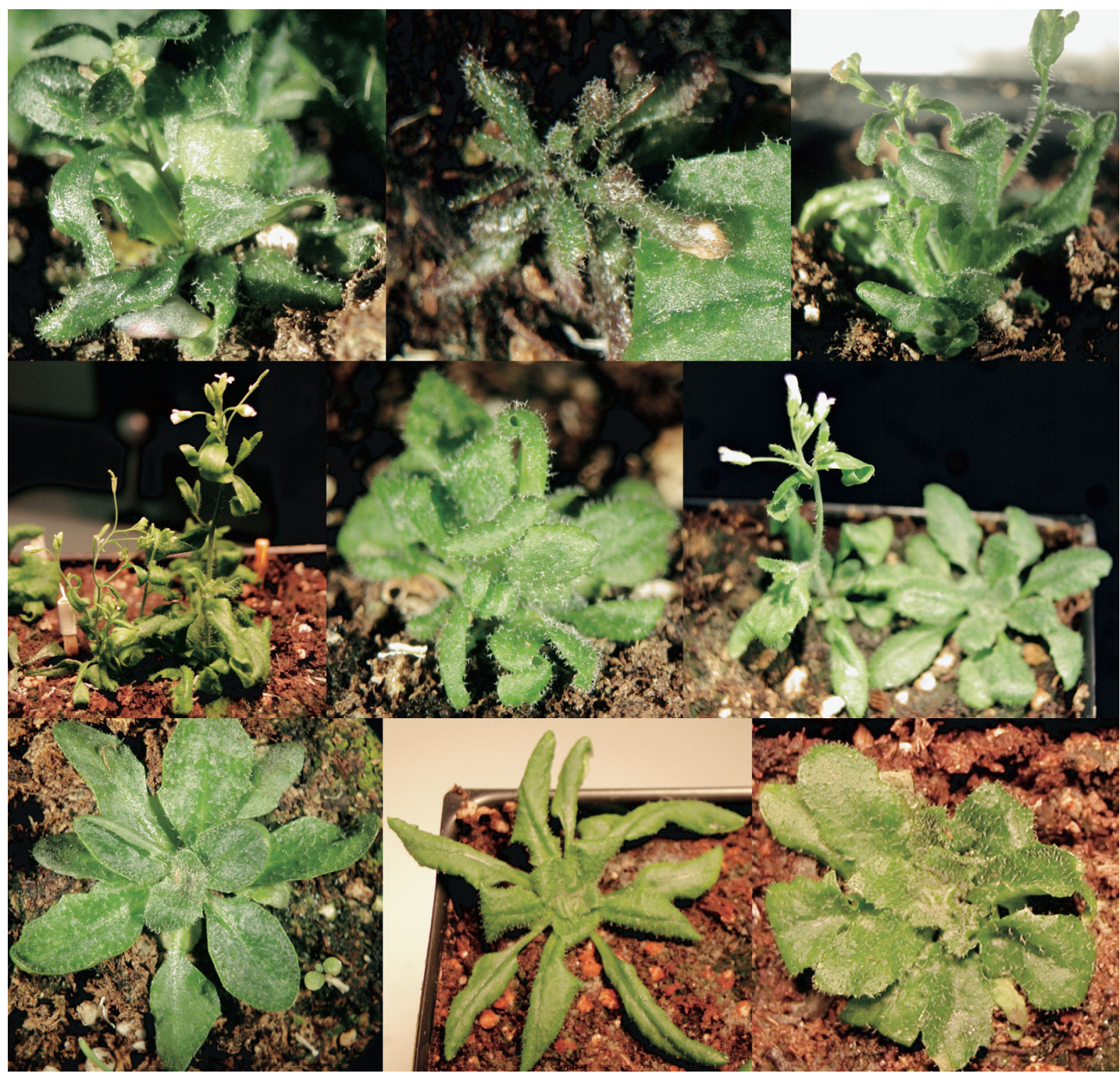

Figure 4. Highly variable developmental phenotypes of $d r m 1 / 2$ met $1 /+$ plants produced through self-pollination of a $d r m 1 / 2$ met $1 /+$ parent.

small RNAs from some additional loci (preferentially from inverted repeats) (Henderson et al. 2006). Moreover, our results as well as several other recent studies unveiled partial but extensive functional redundancy among DCL2, DCL3, and DCL4 in processing a subset of small RNAs (Xie et al. 2004; Gasciolli et al. 2005; Henderson et al. 2006). Finally, we showed that siRNAs generated by DCL3 play a major role in directing DNA methylation, but at some loci, DCL2 and DCL4 products can also assist in this process (Henderson et al. 2006).

The specificity of RNA-directed DNA methylation (RdDM) likely resides in the primary sequences of siRNAs, and the key components that connect DRM1/2 to the RNAi pathway are the ARGONAUTE proteins. In Arabidopsis, AGO4 plays a major role in this process, as the ago 4 mutant displayed reduced non-CG methylation at a number of endogenous loci and failed to de novo methylate a transgene (Zilberman et al. 2003, 2004; Chan et al. 2004). We have recently performed a detailed analysis of AGO4 with regard to its protein stability in several mutant backgrounds, its interaction with other components of the RNAi pathway, and its subnuclear localizations ( $\mathrm{Li}$ et al. 2006; Pontes et al. 2006). Interestingly, AGO4 becomes unstable in the absence of NRPD1a, RDR2, and DCL3, but the loss of NRPD1b or DRM2 does not affect AGO4 protein level. This observation strongly suggested a hierarchical order of action, where NRPD1a, RDR2, and DCL3 function upstream of AGO4 whereas NRPD1b and DRM2 are either at the same step or downstream from AGO4 ( $\mathrm{Li}$ et al. 2006). It is interesting to consider that such a property of AGO4 may offer a "safeguard" for the RdDM process to ensure that, in the absence of siRNAs generated by upstream components, AGO4 would not target spurious DNA methylation to 
inappropriate loci. Further support of the close relationship between AGO4 and NRPD1b came from the observations that they not only colocalize in the nucleus, but also physically interact with each other. Finally and most significantly, AGO4 localizes to the "Cajal body" where the processing and maturation of several ribonucleoprotein complexes take place. Taken together, these results have led us to propose a model where double-stranded RNAs produced by NPRD1a and RDR2 are processed into 24-nucleotide siRNAs by DCL3 in the Cajal body. An AGO4/NRPD1b/siRNA ribonucleoprotein complex is then assembled and relocated to the target locus through sequence homology to the siRNA, and recruits DRM1/2 for DNA methylation ( $\mathrm{Li}$ et al. 2006).

In contrast to DRM1/2, recruitment of CMT3 for CNG methylation depends on the methylation of two lysine residues on the histone $\mathrm{H} 3$ amino-terminal tail: lysine 9 (K9) and lysine 27 (K27). This was initially indicated by the presence of a chromodomain in CMT3 (Lindroth et al. 2001), and experimental evidence came from two studies. First, a histone methyltransferase mutant (kryptonite) affecting $\mathrm{H} 3 \mathrm{~K} 9$ methylation also affected CNG methylation at several endogenous loci (Jackson et al. 2002; Malagnac et al. 2002). Second, CMT3 was found to bind to $\mathrm{H} 3$ peptide methylated at both $\mathrm{K} 9$ and $\mathrm{K} 27$ in vitro, and H3 K27 methylation colocalized with H3 K9 methylation at CMT3-controlled loci (Lindroth et al. 2004). It is conceivable that alterations in $\mathrm{H} 3 \mathrm{~K} 9$ and $\mathrm{K} 27$ methylation could lead to changes in CMT3-dependent CNG and $\mathrm{CHH}$ methylation, thus providing another mechanism by which non-CG DNA methylation can be regulated.

The studies described above identified factors required for non-CG methylation, but they did not address whether non-CG methylation can be reestablished once it is lost from an endogenous locus. Two lines of evidence suggested that such reestablishment can readily take place. First, the phenotype of $d \mathrm{rm} 1 / 2 \mathrm{cmt} 3$ is strictly recessive and co-segregates tightly with the $d r m 1 / 2 \mathrm{cmt} 3$ genotype (Chan et al. 2006). Second, introducing DRM2 or CMT3 as a transgene into the $d \mathrm{rm} 1 / 2 \mathrm{cmt} 3$ mutant restored a wild-type phenotype as well as non-CG methylation at endogenous loci (Chan et al. 2006). These results were in stark contrast to met 1 or $d d m 1$ where the hypomethylated CG sites were generally not remethylated when crossed to wild type (Vongs et al. 1993; Finnegan et al. 1996; Kakutani et al. 1999; Saze et al. 2003). They are also in agreement with the notion that CG methylation is relatively stably maintained, whereas the elimination and reestablishment of non-CG methylation can be subject to developmental regulation.

\section{HIGH-RESOLUTION MAPPING OF DNA METHYLATION IN THE ENTIRE ARABIDOPSIS GENOME}

Extensive genetic, molecular, and genomic analyses in recent years using Arabidopsis as a model system have demonstrated the importance of DNA methylation in suppressing transposons and regulating gene expression (Chan et al. 2005). An accumulating body of data has also broadened our understanding of the establishment and maintenance of DNA methylation as well as its interactions with other epigenetic pathways. However, many important questions had remained unanswered. For example, the extent and distribution of DNA methylation had not been determined on a genome-wide scale, and very few genes were identified as being directly regulated by DNA methylation. This largely reflects technical difficulty in identifying the sites of DNA methylation in a complete eukaryotic genome in a high-throughput manner. We have recently optimized two biochemical methods to separate methylated and unmethylated DNA, followed by hybridization to high-density, whole-genome tiling arrays. This has allowed the genome-wide and highresolution mapping of DNA methylation in Arabidopsis (Zhang et al. 2006).

DNA-methylated regions account for approximately 19\% of the Arabidopsis genome and are highly enriched in centromeric and pericentromeric heterochromatin, where transposable elements and other repetitive sequences cluster. siRNA clusters, but not miRNA-producing or target genes, are also heavily methylated. Unexpectedly, we found that roughly one-third of Arabidopsis genes were methylated in the transcribed regions (body-methylation). Such methylation is biased toward the $3^{\prime}$ end of genes, largely independent of siRNAs and maintained primarily by MET1. The function of genic body-methylation remains unclear, as neither sense nor antisense transcription of body-methylated genes changes systematically when it is lost. In contrast, although the number of genes that contain DNA methylation in their promoters is relatively small, promotermethylation appears to be more important in down-regulating gene expression level. Interestingly, a subset of promoter-methylated genes appear to have very tissue-specific expression patterns.

The vast majority of genomic regions methylated in wild type remains methylated in $d \mathrm{rm} 1 / 2 \mathrm{cmt} 3$; a relatively small number of hypomethylated regions are generally CG-poor. This is consistent with previous results that the loss of non-CG methylation does not significantly disturb CG methylation, and suggests that in most cases non-CG methylation colocalizes with CG methylation. In contrast, only about a third of the methylated regions are still detectable in met1. The residual methylation is highly enriched at repetitive sequences, and is likely maintained by DRM1/2 and CMT3.

Comparison of the genes that were most significantly increased in expression in met 1 and $d r m 1 / 2 \mathrm{cmt} 3$ revealed an important distinction. Most genes that were overexpressed in met 1 were pseudogenes with a pronounced heterochromatic distribution. In contrast, most up-regulated genes (69\%) in $d r m 1 / 2 \mathrm{cmt} 3$ had known functions and were distributed throughout euchromatin. These results provide strong evidence for the role of non-CG methylation in regulating gene expression and identified a host of candidate genes that may be directly regulated by non-CG methylation. Moreover, they are in agreement with the notion discussed earlier that CG methylation is important for the silencing of transposons and the maintenance of heterochromatin, whereas non-CG methylation plays a more central role in regulating developmentally important genes. 


\section{CONCLUSIONS}

In summary, we have presented genetic evidence that $\mathrm{CG}$ and non-CG methylation function in a partially redundant manner to regulate many aspects of the Arabidopsis development. Analyses of the interactions between DNA methylation and other epigenetic pathways such as RNAi and histone methylation provided important clues as to how DNA methylation itself is likely regulated. Finally, the results from the genomic analysis have broadened our understanding of DNA methylation patterns on a genome-wide scale and have identified many candidate genes controlled by DNA methylation.

Future studies should be directed to address several outstanding questions. For example, a large number of genes were found to be misregulated in $\mathrm{met} 1$ or $\mathrm{drm} 1 / \mathrm{drm} 2 \mathrm{cmt} 3$ (Zhang et al. 2006), yet our genetic analysis suggested that many more genes may be controlled redundantly by $\mathrm{CG}$ and non-CG methylation. DNA methylation and expression profiling of mutants such as $d r m 1 / 2$ met1, cmt 3 met 1 , or drm 1/2 cmt3 metl should provide candidates for such genes. Another interesting finding described here is that many promoter-methylated genes are expressed in a tissue-specific manner (Zhang et al. 2006), thus raising the possibility that demethylation may occur at their promoters in specific tissues. Future studies should determine whether a general mechanism exists that removes DNA methylation from these promoters in specific tissues, thus allowing the expression of otherwise silenced genes.

\section{ACKNOWLEDGMENTS}

We thank Dr. Simon Chan for his generous help in constructing DNA methyltransferase mutants, and members of the Jacobsen laboratory for helpful discussions and critical reading of the manuscript. Work in the Jacobsen lab is supported by National Institutes of Health grant GM60398 and a grant from the National Institutes of Health ENCODE Program HG003523. S.E.J. is an investigator of the Howard Hughes Medical Institute.

\section{REFERENCES}

Bartee L., Malagnac F., and Bender J. 2001. Arabidopsis cmt3 chromomethylase mutations block non-CG methylation and silencing of an endogenous gene. Genes Dev. 15: 1753.

Bird A. 2002. DNA methylation patterns and epigenetic memory. Genes Dev. 16: 6.

Bitonti M.B., Cozza R., Chiappetta A., Giannino D., Ruffini Castiglione M., Dewitte W., Mariotti D., Van Onckelen H., and Innocenti A.M. 2002. Distinct nuclear organization, DNA methylation pattern and cytokinin distribution mark juvenile, juvenile-like and adult vegetative apical meristems in peach (Prunus persica (L.) Batsch). J. Exp. Bot. 53: 1047.

Borsani O., Zhu J., Verslues P.E., Sunkar R., and Zhu J.K. 2005. Endogenous siRNAs derived from a pair of natural cis-antisense transcripts regulate salt tolerance in Arabidopsis. Cell 123: 1279.

Cao X. and Jacobsen S.E. 2002a. Locus-specific control of asymmetric and $\mathrm{CpNpG}$ methylation by the DRM and CMT3 methyltransferase genes. Proc. Natl. Acad. Sci. (suppl. 4) 99: 16491.

- 2002b. Role of the Arabidopsis DRM methyltransferases in de novo DNA methylation and gene silencing. Curr. Biol. 12: 1138.
Cao X., Aufsatz W., Zilberman D., Mette M.F., Huang M.S., Matzke M., and Jacobsen S.E. 2003. Role of the DRM and CMT3 methyltransferases in RNA-directed DNA methylation. Curr. Biol. 13: 2212.

Chan S.W., Henderson I.R., and Jacobsen S.E. 2005. Gardening the genome: DNA methylation in Arabidopsis thaliana. Nat. Rev. Genet. 6: 351.

Chan S.W.-L., Henderson I.R., Zhang X., Shah G., Chien J.S.C., and Jacobsen S.E. 2006. RNAi, DRD1 and histone methylation actively target developmentally important non-CG DNA methylation in Arabidopsis. PLoS Genet. 2:e83.

Chan S.W., Zilberman D., Xie Z., Johansen L.K., Carrington J.C., and Jacobsen S.E. 2004. RNA silencing genes control de novo DNA methylation. Science 303: 1336.

Dalmay T., Hamilton A., Rudd S., Angell S., and Baulcombe D.C. 2000. An RNA-dependent RNA polymerase gene in Arabidopsis is required for posttranscriptional gene silencing mediated by a transgene but not by a virus. Cell 101: 543 .

Dunoyer P., Himber C., and Voinnet O. 2005. DICER-LIKE 4 is required for RNA interference and produces the 21nucleotide small interfering RNA component of the plant cellto-cell silencing signal. Nat. Genet. 37: 1356.

Egger G., Jeong S., Escobar S.G., Cortez C.C., Li T.W., Saito Y., Yoo C.B., Jones P.A., and Liang G. 2006. Identification of DNMT1 (DNA methyltransferase 1) hypomorphs in somatic knockouts suggests an essential role for DNMT1 in cell survival. Proc. Natl. Acad. Sci. 103: 14080.

Finnegan E.J., Peacock W.J., and Dennis E.S. 1996. Reduced DNA methylation in Arabidopsis thaliana results in abnormal plant development. Proc. Natl. Acad. Sci. 93: 8449.

Finnegan E.J., Genger R.K., Peacock W.J., and Dennis E.S. 1998. DNA methylation in plants. Annu. Rev. Plant Physiol. Plant Mol. Biol. 49: 223.

Fraga M.F., Canal M.J., and Rodriguez R. 2002. Phase-change related epigenetic and physiological changes in Pinus radiata D. Don. Planta 215: 672 .

Gasciolli V., Mallory A.C., Bartel D.P., and Vaucheret H. 2005. Partially redundant functions of Arabidopsis DICER-like enzymes and a role for DCL4 in producing trans-acting siRNAs. Curr. Biol. 15: 1494.

Goll M.G. and Bestor T.H. 2005. Eukaryotic cytosine methyltransferases. Annu. Rev. Biochem. 74: 481.

Hamilton A., Voinnet O., Chappell L., and Baulcombe D. 2002. Two classes of short interfering RNA in RNA silencing. EMBO J. 21: 4671.

Henderson I.R., Zhang X., Lu C., Johnson L., Meyers B.C., Green P.J., and Jacobsen S.E. 2006. Dissecting Arabidopsis thaliana DICER function in small RNA processing, gene silencing and DNA methylation patterning. Nat. Genet. 38: 721.

Herr A.J., Jensen M.B., Dalmay T., and Baulcombe D.C. 2005. RNA polymerase IV directs silencing of endogenous DNA. Science 308: 118 .

Jackson J.P., Lindroth A.M., Cao X., and Jacobsen S.E. 2002. Control of CpNpG DNA methylation by the KRYPTONITE histone H3 methyltransferase. Nature 416: 556.

Jacobsen S.E. and Meyerowitz E.M. 1997. Hypermethylated SUPERMAN epigenetic alleles in Arabidopsis. Science 277: 1100.

Jeddeloh J.A., Stokes T.L., and Richards E.J. 1999. Maintenance of genomic methylation requires a SWI2/SNF2-like protein. Nat. Genet. 22: 94.

Kakutani T., Munakata K., Richards E.J., and Hirochika H. 1999. Meiotically and mitotically stable inheritance of DNA hypomethylation induced by $d d m 1$ mutation of Arabidopsis thaliana. Genetics 151: 831 .

Kankel M.W., Ramsey D.E., Stokes T.L., Flowers S.K., Haag J.R., Jeddeloh J.A., Riddle N.C., Verbsky M.L., and Richards E.J. 2003. Arabidopsis MET1 cytosine methyltransferase mutants. Genetics 163: 1109.

Kanno T., Huettel B., Mette M.F., Aufsatz W., Jaligot E., Daxinger L., Kreil D.P., Matzke M., and Matzke A.J. 2005. Atypical RNA polymerase subunits required for RNAdirected DNA methylation. Nat. Genet. 37: 761. 
Li C.F., Pontes O., El-Shami M., Henderson I.R., Bernatavichute Y.V., Chan S.W., Lagrange T., Pikaard C.S., and Jacobsen S.E. 2006. An ARGONAUTE4-containing nuclear processing center colocalized with Cajal bodies in Arabidopsis thaliana. Cell 126: 93.

Lindroth A.M., Cao X., Jackson J.P., Zilberman D., McCallum C.M., Henikoff S., and Jacobsen S.E. 2001. Requirement of CHROMOMETHYLASE3 for maintenance of CpXpG methylation. Science 292: 2077.

Lindroth A.M., Shultis D., Jasencakova Z., Fuchs J., Johnson L., Schubert D., Patnaik D., Pradhan S., Goodrich J., Schubert I., et al. 2004. Dual histone H3 methylation marks at lysines 9 and 27 required for interaction with CHROMOMETHYLASE3. EMBO J. 23: 4286.

Lu C., Tej S.S., Luo S., Haudenschild C.D., Meyers B.C., and Green P.J. 2005. Elucidation of the small RNA component of the transcriptome. Science 309: 1567.

Malagnac F., Bartee L., and Bender J. 2002. An Arabidopsis SET domain protein required for maintenance but not establishment of DNA methylation. EMBO J. 21: 6842.

Martienssen R.A. and Colot V. 2001. DNA methylation and epigenetic inheritance in plants and filamentous fungi. Science 293: 1070 .

Messeguer R., Ganal M.W., Steffens J.C., and Tanksley S.D. 1991. Characterization of the level, target sites and inheritance of cytosine methylation in tomato nuclear DNA. Plant Mol. Biol. 16: 753.

Onodera Y., Haag J.R., Ream T., Nunes P.C., Pontes O., and Pikaard C.S. 2005. Plant nuclear RNA polymerase IV mediates siRNA and DNA methylation-dependent heterochromatin formation. Cell 120: 613 .

Pontes O., Li C.F., Nunes P.C., Haag J., Ream T., Vitins A., Jacobsen S.E., and Pikaard C.S. 2006. The Arabidopsis chromatin-modifying nuclear siRNA pathway involves a nucleolar RNA processing center. Cell 126: 79.

Pontier D., Yahubyan G., Vega D., Bulski A., Saez-Vasquez J., Hakimi M.A., Lerbs-Mache S., Colot V., and Lagrange T. 2005. Reinforcement of silencing at transposons and highly repeated sequences requires the concerted action of two distinct RNA polymerases IV in Arabidopsis. Genes Dev. 19: 2030.

Ronemus M.J., Galbiati M., Ticknor C., Chen J., and Dellaporta S.L. 1996. Demethylation-induced developmental pleiotropy in Arabidopsis. Science 273: 654.

Ruiz-Garcia L., Cervera M.T., and Martinez-Zapater J.M. 2005. DNA methylation increases throughout Arabidopsis development. Planta 222: 301
Saze H., Scheid O.M., and Paszkowski J. 2003. Maintenance of $\mathrm{CpG}$ methylation is essential for epigenetic inheritance during plant gametogenesis. Nat. Genet. 34: 65.

Schauer S.E., Jacobsen S.E., Meinke D.W., and Ray A. 2002. DICER-LIKE1: Blind men and elephants in Arabidopsis development. Trends Plant Sci. 7: 487.

Soppe W.J., Jacobsen S.E., Alonso-Blanco C., Jackson J.P., Kakutani T., Koornneef M., and Peeters A.J. 2000. The late flowering phenotype of fwa mutants is caused by gain-offunction epigenetic alleles of a homeodomain gene. Mol. Cell 6: 791.

Vongs A., Kakutani T., Martienssen R.A., and Richards E.J. 1993. Arabidopsis thaliana DNA methylation mutants. Science 260: 1926.

Xie Z., Allen E., Wilken A., and Carrington J.C. 2005. DICERLIKE 4 functions in trans-acting small interfering RNA biogenesis and vegetative phase change in Arabidopsis thaliana. Proc. Natl. Acad. Sci. 102: 12984.

Xie Z., Johansen L.K., Gustafson A.M., Kasschau K.D., Lellis A.D., Zilberman D., Jacobsen S.E., and Carrington J.C. 2004. Genetic and functional diversification of small RNA pathways in plants. PLoS Biol. 2: E104.

Xiong L.Z., Xu C.G., Saghai Maroof M.A., and Zhang Q. 1999. Patterns of cytosine methylation in an elite rice hybrid and its parental lines, detected by a methylation-sensitive amplification polymorphism technique. Mol. Gen. Genet. 261: 439.

Yoder J.A., Walsh C.P., and Bestor T.H. 1997. Cytosine methylation and the ecology of intragenomic parasites. Trends Genet. 13: 335.

Yoshikawa M., Peragine A., Park M.Y., and Poethig R.S. 2005. A pathway for the biogenesis of trans-acting siRNAs in Arabidopsis. Genes Dev. 19: 2164.

Zhang X., Yazaki J., Sundaresan A., Cokus S., Chan S.W., Chen H., Henderson I.R., Shinn P., Pellegrini M., Jacobsen S.E., and Ecker J.R. 2006. Genome-wide high-resolution mapping and functional analysis of DNA methylation in Arabidopsis. Cell 126: 1189.

Zilberman D., Cao X., and Jacobsen S.E. 2003. ARGONAUTE4 control of locus-specific siRNA accumulation and DNA and histone methylation. Science 299: 716 .

Zilberman D., Cao X., Johansen L.K., Xie Z., Carrington J.C., and Jacobsen S.E. 2004. Role of Arabidopsis ARGONAUTE4 in RNA-directed DNA methylation triggered by inverted repeats. Curr. Biol. 14: 1214.

Zluvova J., Janousek B., and Vyskot B. 2001. Immunohistochemical study of DNA methylation dynamics during plant development. J. Exp. Bot. 52: 2265. 


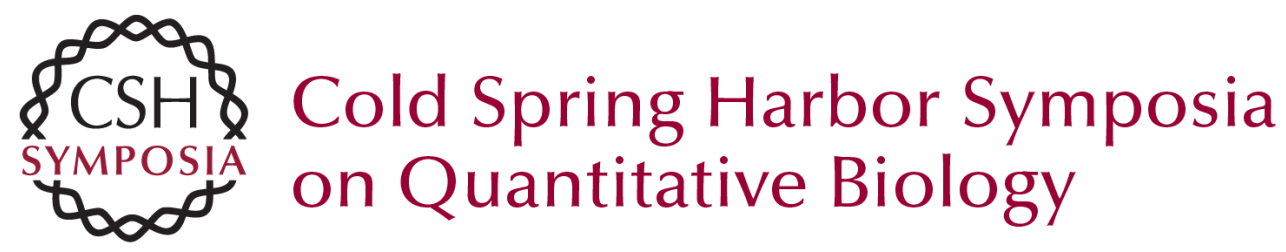

\section{Genetic Analyses of DNA Methyltransferases in Arabidopsis thaliana}

X. ZHANG and S.E. JACOBSEN

Cold Spring Harb Symp Quant Biol 2006 71: 439-447

Access the most recent version at doi:10.1101/sqb.2006.71.047

References This article cites 51 articles, 21 of which can be accessed free at: http://symposium.cshlp.org/content/71/439.full.html\#ref-list-1

License

Email Alerting Receive free email alerts when new articles cite this article - sign up in Service the box at the top right corner of the article or click here. 